\title{
On Rectifying Curves and Their Characterization in Lorentz $n$-Space
}

\author{
Tunahan Turhan* \\ (Communicated by Kazım İlarslan)
}

\begin{abstract}
This paper deals with rectifying curves and their characterization in arbitrary dimensional Lorentz $n$-space. Considering the structure of a rectifying curve, we give some generalizations of such curves in Lorentz $n$-space. Moreover, we characterize and prove some properties of these curves in terms of their curvature functions.
\end{abstract}

Keywords: Curvature; Frenet frame; Lorentz n-space; null rectifying curve; spacelike rectifying curve; timelike rectifying curve.

AMS Subject Classification (2010): Primary: $53 A 35$; Secondary: $53 B 30$

\section{Introduction}

The definition and characterization of rectifying curves has started by the work of [3], in which the author determined all rectifying curves in $\mathbb{E}^{3}$. Rectifying curves are space curves, where position vector always lies in its rectifying plane. The rectifying plane is spanned by tangent vector $T(s)$ and the binormal vector $B(s)$. So, with respect to some chosen origin, the position vector of a rectifying curve $\alpha$ satisfies the equation

$$
\alpha(s)=\lambda(s) T(s)+\mu(s) B(s),
$$

where $\lambda(s)$ and $\mu(s)$ are arbitrary differentiable functions, [3].

Euclidean rectifying curves have an important role in kinematic. For example, the position vector of a rectifying curve is always in the direction of the Darboux vector. Therefore, rectifying curves can be interpreted kinematically as those curves whose position vector field determines the axis of instantaneous rotation at each point of the curve, [3]. Also, Euclidean rectifying curves have interesting geometric properties. So, such curves are studied by many researchers, such as [2, 3, 4, 6, 11, 15]. For example, the authors in [6] defined the rectifying curves in Euclidean 4-space. Later on, in [2], the authors extended the results in [6], to the general case $\mathbb{E}^{n}$. Then, the definition and concept of a rectifying curve is extended to Lorentz-Minkowski space. The presence of the casual character of curves and vectors in Lorentz-Minkowski space often causes important and interesting differences between Lorentzian and Riemannian geometry. So, there are many studies about the rectifying curves in Lorentz-Minkowski space, $[5,7,8,9]$. Some characterizations of spacelike, timelike and null rectifying curves in Minkowski 3-space are given in [9]. The characterization of null, pseudo null and partially null rectifying curves in Minkowski space-time are studied in [5]. Also, the authors in [1] defined a spacelike rectifying curve in $\mathbb{E}_{1}^{4}$. The definition of the rectifying curve in other spaces such as three dimensional sphere, [12], and pseudo-Galilean space, [14], can be found in the literature.

In the current study, we want to contribute to the study of null and spacelike rectifying curves and present some results for such curves in the Lorentz $n$-space $\mathbb{E}_{1}^{n}$. Firstly, we recall some preliminaries about the theory of curves in $\mathbb{E}_{1}^{n}$. After that, we examine null rectifying curves in $\mathbb{E}_{1}^{n}$. Then, we study spacelike rectifying curves in $\mathbb{E}_{1}^{n}$ and prove some properties and characterizations of these curves. 


\section{Preliminaries}

We first recall some general notions and notations needed throughout the paper, and repeat some of the definitions mentioned in the introduction section, more formally.

Let $E_{1}^{n}$ indicate the $n$-dimensional Lorentz space. For vectors $x=\left(x_{1}, x_{2}, \ldots, x_{n}\right)$ and $y=\left(y_{1}, y_{2}, \ldots, y_{n}\right)$, the metric we use in this paper is

$$
\langle x, y\rangle=-x_{1} y_{1}+\sum_{i=2}^{n} x_{i} y_{i} .
$$

The vector $v \in \mathbb{E}_{1}^{n}$ is called spacelike, null (lightlike) or timelike if $\langle v, v\rangle>0$ or $v=0,\langle v, v\rangle=0$ and $v \neq 0$, and $\langle v, v\rangle<0$, respectively.

We define the signature of a vector $v$ as

$$
\epsilon= \begin{cases}1 & , v \text { is spacelike } \\ 0 & , v \text { is null (lightlike) }, \\ -1 & , v \text { is timelike. }\end{cases}
$$

The norm of vector $v \in \mathbb{E}_{1}^{n}$ is defined by $\|v\|=\sqrt{|\langle v, v\rangle|}$.

A curve $\alpha$ in $E_{1}^{n}$ is said to be spacelike if all of its velocity vectors $\alpha^{\prime}$ are spacelike, it is similar for timelike and null, [13].

Let $\alpha: I \subset \mathbb{R} \longrightarrow E_{1}^{n}$ be an arclength parametrized spacelike curve. The Frenet equations of the spacelike curve $\alpha$ are as follows [10]:

$$
\begin{aligned}
T^{\prime}(s) & =\kappa_{1} N(s), \\
N^{\prime}(s) & =-\epsilon_{1} \kappa_{1} T(s)+\kappa_{2} B_{1}(s), \\
B_{1}^{\prime}(s) & =-\epsilon_{1} \epsilon_{2} \kappa_{2} N(s)+\kappa_{3} B_{2}(s), \\
& \vdots \\
B_{i}^{\prime}(s) & =-\epsilon_{i} \epsilon_{i+1} \kappa_{i+1} B_{i-1}(s)+\kappa_{i+2} B_{i+1}(s), \\
& \vdots \\
B_{n-2}^{\prime}(s) & =-\epsilon_{n-2} \epsilon_{n-1} \kappa_{n-1} B_{n-3}(s),
\end{aligned}
$$

where $T, N, B_{1}, \ldots, B_{n-2}$ are mutually orthogonal vectors and $\kappa_{1}, \kappa_{2}, \ldots, \kappa_{i}, \ldots, \kappa_{n-1}$ are the curvatures and $\epsilon_{1}, \epsilon_{2}, \ldots, \epsilon_{i}, \ldots \epsilon_{n-1}$ are the signatures of $\left\{N, B_{1}, \ldots, B_{n-2}\right\}$. Therefore, $\left\{T, N, B_{1}, \ldots, B_{n-2}\right\}$ is an orthonormal frame of $\alpha$.

Definition 2.1. A curve $\alpha: I \rightarrow \mathbb{E}_{1}^{n}$ is called rectifying curve if for all $s \in I$, the orthogonal complement of $N(s)$ contains a fixed point.

Since the orthogonal complement of $N$ is $N^{\perp}=\left\{\mathrm{v} \in T_{\alpha} \mathbb{E}_{1}^{n} \mid\langle\mathrm{v}, N\rangle=0\right\}$, the position vector of a spacelike rectifying curve $\alpha$ in $\mathbb{E}_{1}^{n}$ can be written as

$$
\alpha=\lambda T+\mu_{1} B_{1}+\ldots+\mu_{n-2} B_{n-2},
$$

where $\lambda, \mu_{1}, \ldots, \mu_{n-2}$ are real functions, [2].

\section{Null Rectifying Curves in $\mathbb{E}_{1}^{n}$}

In this section, we give some generalizations of the null rectifying curves in $\mathbb{E}_{1}^{n}, n>4$. Let $\alpha: I \subset \mathbb{R} \rightarrow \mathbb{E}_{1}^{n}$ be a null curve parametrized by the pseudo-arclength such that $\left\{\alpha^{\prime}(t), \ldots, \alpha^{n}(t)\right\}$ is a basis of $T_{\alpha(t)} \mathbb{E}_{1}^{n}$ for all $t$. Then there exists only one Frenet frame satisfying the equations

$$
\begin{aligned}
T^{\prime} & =N \\
B_{1}^{\prime} & =-\kappa_{1} N+\kappa_{2} B_{2} \\
N^{\prime} & =\kappa_{1} T-B_{1}, \\
B_{2}^{\prime} & =-\kappa_{2} T+\kappa_{3} B_{3}, \\
B_{i}^{\prime} & =-\kappa_{i} B_{i-1}+\kappa_{i+1} B_{i+1}, \quad i \in\{3, \ldots, n-3\}, \\
B_{n-2}^{\prime} & =-\kappa_{n-2} B_{n-3},
\end{aligned}
$$


where

$$
\langle T, T\rangle=\left\langle B_{1}, B_{1}\right\rangle=0, \quad\left\langle T, B_{1}\right\rangle=1
$$

and

$$
\langle N, N\rangle=\left\langle B_{2}, B_{2}\right\rangle=\left\langle B_{3}, B_{3}\right\rangle=\ldots=\left\langle B_{n-2}, B_{n-2}\right\rangle=1 .
$$

Let $\alpha(s)$ be a null rectifying curve in $\mathbb{E}_{1}^{n}$, parametrized by pseudo-arclength $s$. Then its position vector satisfies the equation

$$
\alpha(s)=\lambda(s) T+\mu_{1}(s) B_{1}+\mu_{2}(s) B_{2}+\ldots+\mu_{n-3}(s) B_{n-3}+\mu_{n-2}(s) B_{n-2},
$$

for some differentiable functions $\lambda(s), \mu_{1}(s), \mu_{2}(s), \ldots, \mu_{n-2}(s)$.

Differentiating (3.2) with respect to $s$ and by using (3.1), we obtain the system of equations

$$
\begin{aligned}
\mu_{1}^{\prime} & =0, \\
\lambda^{\prime}-\mu_{2} \kappa_{2} & =1, \\
\lambda-\mu_{1} \kappa_{1} & =0, \\
\mu_{i}^{\prime}+\mu_{i-1} \kappa_{i}-\mu_{i+1} \kappa_{i+1} & =0, \\
\mu_{n-2}^{\prime}+\mu_{n-3} \kappa_{n-3} & =0 .
\end{aligned}
$$

From equation (3.2), we get that, the tangential component of the position vector of $\alpha$ is given by $\langle\alpha, T\rangle=$ $\mu_{1}(s)$. From equation (3.3), we obtain $\mu_{1}(s)=c, c \in \mathbb{R}$. Considering the case $\langle\alpha, T\rangle=0$, we get the following theorems.

Theorem 3.1. Let $\alpha(s)$ be a null rectifying curve in $\mathbb{E}_{1}^{n}$, parametrized by pseudo-arclength $s$. If $\alpha$ is a rectifying curve with the tangential component $\langle\alpha, T\rangle=0$, then the following statements hold:

[i] The components of the position vector of $\alpha$ are

$$
\lambda=0, \quad \mu_{1}=0
$$

and

$$
\mu_{i}(s)=\sum_{k=0}^{i-2} \mu_{i, k}(s) \frac{\partial^{k}}{\partial s^{k}}\left(-\frac{1}{\kappa_{2}(s)}\right), \quad i \in\{2,3, \ldots, n-3\},
$$

where the functions $\mu_{i, k}$ are inductively defined by the following system:

$$
\begin{aligned}
& \mu_{1,0}(s)=0, \\
& \mu_{2,0}(s)=1,
\end{aligned}
$$

and for $i \in\{3,4, \ldots, n-2\}$,

$$
\begin{aligned}
\mu_{i, 0}(s) & =\frac{\kappa_{i-1}(s) \mu_{i-2,0}(s)+\mu_{i-1,0}^{\prime}(s)}{\kappa_{i}(s)}, \\
\mu_{i, k}(s) & =\frac{\kappa_{i-1}(s) \mu_{i-2, k}(s)+\mu_{i-1, k}^{\prime}(s)+\mu_{i-1, k}(s)}{\kappa_{i}(s)}, \quad k \in\{1,2, \ldots, i-4\} \\
\mu_{i, i-3} & =\frac{\mu_{i-1, i-4}(s)+\mu_{i-1, i-3}^{\prime}(s)}{\kappa_{i}(s)}, \\
\mu_{i, i-2} & =\frac{\mu_{i-1, i-3}(s)}{\kappa_{i}(s)} .
\end{aligned}
$$

[ii] If $\kappa_{2}, \kappa_{3}, \ldots, \kappa_{n-1}$ are constants but non-zero, then $\alpha$ lies in pseudosphere $S_{1}^{n-1}(r), r \in \mathbb{R}_{0}^{+}$.

Proof. Let $\alpha(s)$ be a null rectifying curve in $\mathbb{E}_{1}^{n}$, parametrized by pseudo-arclength $s$ and $\langle\alpha, T\rangle=0$. Then its position vector is given by (3.2). So, the system of the equations (3.3)-(3.7) reduces to

$$
\begin{gathered}
\mu_{1}=0, \quad \lambda=0, \\
\mu_{2}=-\frac{1}{\kappa_{2}} .
\end{gathered}
$$


Introducing functions $\mu_{1,0}$ and $\mu_{2,0}$, one rewrites these equations as

$$
\mu_{1}(s)=\mu_{1,0}(s)\left(-\frac{1}{\kappa_{1}}\right)
$$

and

$$
\mu_{2}(s)=\mu_{2,0}(s)\left(-\frac{1}{\kappa_{1}}\right)
$$

where $\mu_{1,0}=0$ and $\mu_{2,0}=1$.

By induction, from equation (3.6),

$$
\mu_{i+1}=\frac{1}{\kappa_{i+1}}\left(\mu_{i}^{\prime}+\mu_{i-1} \kappa_{i}\right)
$$

we obtain

$$
\mu_{i}(s)=\sum_{k=0}^{i-2} \mu_{i, k}(s) \frac{\partial^{k}}{\partial s^{k}}\left(-\frac{1}{\kappa_{2}(s)}\right),
$$

for $i \in\{2,3, \ldots, n-3\}$. Here the functions $\mu_{i, k}$ are defined by system (3.8). This proves statement $[i]$.

If the curvatures $\kappa_{2}, \ldots, \kappa_{n-1}$ are constants, the components of the position vector of $\alpha, \mu_{2}, \mu_{3}, \ldots, \mu_{n-2}$ are constant numbers. By relations $\mu_{1}=0$ and $\lambda=0$, we obtain that $\alpha$ has equation

$$
\alpha(s)=\mu_{2} B_{2}(s)+\mu_{3} B_{3}(s)+\ldots+\mu_{n-2} B_{n-2}(s) .
$$

From the last equation, we get

$$
\langle\alpha(s), \alpha(s)\rangle=\mu_{2}^{2}+\mu_{3}^{2}+\ldots+\mu_{n-2}^{2}=r^{2}, \quad r \in \mathbb{R}_{0}^{+},
$$

which means that $\alpha$ lies in pseudosphere $S_{1}^{n-1}(r)$ with center at the origin and radius $r$. This proves statement $[i i]$.

Theorem 3.2. Let $\alpha(s)$ be a null curve in $\mathbb{E}_{1}^{n}$ with non-zero curvatures. Then $\alpha$ is congruent to a null rectifying curve if and only if

$$
\kappa_{n-3} \sum_{k=0}^{n-5} \mu_{n-3, k}(s) \frac{\partial^{k}}{\partial s^{k}}\left(-\frac{1}{\kappa_{2}(s)}\right)+\sum_{k=0}^{n-4}\left(\mu_{n-2, k}(s) \frac{\partial^{k}}{\partial s^{k}}\left(-\frac{1}{\kappa_{2}(s)}\right)\right)^{\prime}=0
$$

where the functions $\mu_{i, k}$ are defined by system (3.8).

Proof. If $\alpha$ is a null rectifying curve, we can use equation (3.7) in equation (3.10). Then we obtain equation (3.11).

Conversely, assume that equation (3.11) is satisfied. Define the curve

$$
\beta(s)=\alpha(s)-\mu_{2}(s) B_{2}(s)-\mu_{3}(s) B_{3}(s)-\ldots-\mu_{n-2}(s) B_{n-2}(s)
$$

with the functions $\mu_{2}(s), \ldots, \mu_{n-2}(s)$ as in equation (3.9) and (3.10). Since $\beta^{\prime}(s)=0$, we can say that $\alpha$ is congruent to a null rectifying curve.

\section{Spacelike Rectifying Curves in $\mathbb{E}_{1}^{n}$}

This section contains some generelizations of the spacelike rectifying curves in $\mathbb{E}_{1}^{n}$.

\subsection{The curvatures of the spacelike rectifying curve}

Differentiating the position vector of $\alpha$ with respect to the arclenght parameter $s$, we get

$$
\alpha^{\prime}(s)=\lambda^{\prime}(s) T(s)+\lambda(s) T^{\prime}(s)+\sum_{i=1}^{n-2}\left(\mu_{i}^{\prime}(s) B_{i}(s)+\mu_{i}(s) B_{i}^{\prime}(s)\right) .
$$


With the help of the Frenet equations in (2.1), we come across with

$$
\begin{aligned}
T=\lambda^{\prime} T & +\left(\lambda \kappa_{1}-\epsilon_{1} \epsilon_{2} \mu_{1} \kappa_{2}\right) N+\left(\mu_{1}^{\prime}-\epsilon_{2} \epsilon_{3} \mu_{2} \kappa_{3}\right) B_{1} \\
& +\sum_{i=2}^{n-3}\left(\mu_{i}^{\prime}+\mu_{i-1} \kappa_{i+1}-\epsilon_{i+1} \epsilon_{i+2} \mu_{i+1} \kappa_{i+2}\right) B_{i}+\left(\mu_{n-2}^{\prime}+\mu_{n-3} \kappa_{n-1}\right) B_{n-2} .
\end{aligned}
$$

So, we obtain

$$
\begin{aligned}
\lambda^{\prime} & =1, \\
\lambda \kappa_{1}-\epsilon_{1} \epsilon_{2} \mu_{1} \kappa_{2} & =0, \\
\mu_{1}^{\prime}-\epsilon_{2} \epsilon_{3} \mu_{2} \kappa_{3} & =0, \\
\mu_{i}^{\prime}+\mu_{i-1} \kappa_{i+1}-\epsilon_{i+1} \epsilon_{i+2} \mu_{i+1} \kappa_{i+2} & =0, \\
\mu_{n-2}^{\prime}+\mu_{n-3} \kappa_{n-1} & =0 .
\end{aligned}
$$

From (4.1), (4.2) and (4.3), we get

$$
\begin{gathered}
\lambda=s+c, \quad c \in \mathbb{R} \\
\mu_{1}=\frac{1}{\epsilon_{1} \epsilon_{2}} \frac{\kappa_{1}}{\kappa_{2}} \lambda, \\
\mu_{2}=\frac{1}{\epsilon_{2} \epsilon_{3}} \frac{1}{\epsilon_{1} \epsilon_{2}}\left(\frac{1}{\kappa_{3}} \frac{\kappa_{1}}{\kappa_{2}}+\frac{\lambda}{\kappa_{3}}\left(\frac{\kappa_{1}}{\kappa_{2}}\right)^{\prime}\right) .
\end{gathered}
$$

Supposing the functions as below

$$
\begin{aligned}
& \mu_{1,0}=\frac{1}{\epsilon_{1} \epsilon_{2}} \lambda, \\
& \mu_{2,0}=\frac{1}{\epsilon_{2} \epsilon_{3}} \frac{1}{\epsilon_{1} \epsilon_{2}} \frac{1}{\kappa_{3}}, \\
& \mu_{2,1}=\frac{1}{\epsilon_{2} \epsilon_{3}} \frac{1}{\epsilon_{1} \epsilon_{2}} \frac{\lambda}{\kappa_{3}},
\end{aligned}
$$

we can rewrite equations (4.7) and (4.8) as follows:

$$
\begin{array}{r}
\mu_{1}=\mu_{1,0} \frac{\kappa_{1}}{\kappa_{2}}, \\
\mu_{2}=\mu_{2,0} \frac{\kappa_{1}}{\kappa_{2}}+\mu_{2,1}\left(\frac{\kappa_{1}}{\kappa_{2}}\right)^{\prime} .
\end{array}
$$

From (4.4) and using equations (4.9) and (4.10), we conclude

$$
\mu_{i}=\sum_{k=0}^{i-1} \mu_{i, k} \frac{\partial^{k}}{\partial s^{k}}\left(\frac{\kappa_{1}}{\kappa_{2}}\right),
$$

for $i=\{3,4, \ldots, n-2\}$.

The system of the functions $\mu_{i, k}$ can be constitued as below:

$$
\begin{aligned}
\mu_{1,0} & =\frac{1}{\epsilon_{1} \epsilon_{2}}(s+c) \\
\mu_{2,0} & =\frac{1}{\epsilon_{2} \epsilon_{3}} \frac{1}{\epsilon_{1} \epsilon_{2}} \frac{1}{\kappa_{3}} \\
\mu_{2,1} & =\frac{1}{\epsilon_{2} \epsilon_{3}} \frac{1}{\epsilon_{1} \epsilon_{2}} \frac{(s+c)}{\kappa_{3}}, \\
\mu_{i, 0} & =\frac{\kappa_{i} \mu_{i-2,0}+\mu_{i-1,0}^{\prime}}{\epsilon_{i} \epsilon_{i+1} \kappa_{i+1}}, \\
\mu_{i, k} & =\frac{\kappa_{i} \mu_{i-2, k}+\mu_{i-1, k}^{\prime}+\mu_{i-1, k-1}}{\epsilon_{i} \epsilon_{i+1} \kappa_{i+1}}, \\
\mu_{i, i-2} & =\frac{\mu_{i-1, i-3}+\mu_{i-1, i-2}^{\prime}}{\epsilon_{i} \epsilon_{i+1} \kappa_{i+1}}, \\
\mu_{i, i-1} & =\frac{\mu_{i-1, i-2}}{\epsilon_{i} \epsilon_{i+1} \kappa_{i+1}},
\end{aligned}
$$


where $k \in\{1,2, \ldots, i-3\}$ and $i \in\{3,4, \ldots, n-2\}$.

Theorem 4.1. Let $\alpha: I \rightarrow \mathbb{E}_{1}^{n}$ be an arclength parametrized curve with non-zero curvatures. Then, $\alpha$ is congruent to a spacelike rectifying curve if and only if

$$
\kappa_{n-1} \sum_{k=0}^{n-4} \mu_{n-3, k} \frac{\partial^{k}}{\partial s^{k}}\left(\frac{\kappa_{1}}{\kappa_{2}}\right)+\sum_{k=0}^{n-3}\left(\mu_{n-2, k} \frac{\partial^{k}}{\partial s^{k}}\left(\frac{\kappa_{1}}{\kappa_{2}}\right)\right)^{\prime}=0,
$$

where $\mu_{i, k}$ is defined in system (4.12).

Proof. The proof of this theorem is trivial by reading the paper in [2].

Theorem 4.2. There exists no spacelike rectifying curve in $\mathbb{E}_{1}^{n}$ with non-zero constant curvatures.

Proof. Suppose that, there exists a spacelike rectifying curve with non-zero constant curvatures. From (4.6), (4.7), (4.8) and (4.4), we find

$$
\mu_{i+1}=\frac{\mu_{i-1} \kappa_{i+1}+\mu_{i}^{\prime}}{\epsilon_{i+1} \epsilon_{i+2} \kappa_{i+2}},
$$

for $i \in\{2,3, \ldots, n-3\}$. With the help of the induction, we get

$$
\begin{aligned}
\mu_{2 m-1} & =\frac{1}{\epsilon_{1} \epsilon_{2} \ldots \epsilon_{2 m}} \frac{\kappa_{1} \kappa_{3} \ldots \kappa_{2 m-1}}{\kappa_{2} \kappa_{4} \ldots \kappa_{2 m}}(s+c), \\
\mu_{2 m} & =\frac{1}{\epsilon_{1} \epsilon_{2} \ldots \epsilon_{2 m+1}} \frac{\sum_{j=1}^{m}\left[\left(\prod_{i=1}^{j} \kappa_{2 i-1} \prod_{i=j+1}^{m} \kappa_{2 i}\right)^{2} \epsilon_{2 j}\right]}{\kappa_{1} \kappa_{2} \kappa_{3} \ldots \kappa_{2 m+1}} .
\end{aligned}
$$

It is clear that, these equations are valid for $m=1$. Putting $m=M+1$ in (4.14), equation (4.16) is generated: If equations (4.14) and (4.15) are valid for $m \in\{1,2, \ldots, M\}$, then

$$
\begin{aligned}
\mu_{2 M+1} & =\frac{\mu_{2 M-1} \kappa_{2 M+1}+\mu_{2 M}^{\prime}}{\epsilon_{2 M+1} \epsilon_{2 M+2} \kappa_{2 M+2}} \\
& =\frac{1}{\epsilon_{1} \epsilon_{2} \ldots \epsilon_{2 M} \epsilon_{2 M+1} \epsilon_{2 M+2}} \frac{\kappa_{1} \kappa_{3} \ldots \kappa_{2 M-1} \kappa_{2 M+1}}{\kappa_{2} \kappa_{4} \ldots \kappa_{2 M} \kappa_{2 M+2}}(s+c) .
\end{aligned}
$$

Putting $m=M+1$ in (4.15), equation (4.17) is generated:

$$
\begin{aligned}
\mu_{2 M+2} & =\frac{\mu_{2 M} \kappa_{2 M+2}+\mu_{2 M+1}^{\prime}}{\epsilon_{2 M+2} \epsilon_{2 M+3} \kappa_{2 M+3}} \\
& =\frac{1}{\epsilon_{1} \epsilon_{2} \ldots \epsilon_{2 M+3}} \frac{\sum_{j=1}^{M+1}\left[\left(\prod_{i=1}^{j} \kappa_{2 i-1} \prod_{i=j+1}^{M+1} \kappa_{2 i}\right)^{2} \epsilon_{2 j}\right]}{\kappa_{1} \kappa_{2} \kappa_{3} \ldots \kappa_{2 M+1} \kappa_{2 M+2} \kappa_{2 M+3}} .
\end{aligned}
$$

For even $n$, from (4.14) and (4.15), equation (4.5) reduces to

$$
\frac{1}{\epsilon_{1} \epsilon_{2} \ldots \epsilon_{2 n-2}} \frac{\kappa_{1} \kappa_{3} \ldots \kappa_{n-3}}{\kappa_{2} \kappa_{4} \ldots \kappa_{n-2}}(s+c) \kappa_{n-1}=0
$$

Since we suppose all the curvatures to be non-zero, a contradiction occurs with (4.18).

For odd $n$, from (4.14) and (4.15), equation (4.5) is rewritten as follows

$$
\frac{1}{\epsilon_{1} \epsilon_{2} \ldots \epsilon_{n-2}} \frac{\sum_{j=1}^{\frac{n-3}{2}}\left[\left(\prod_{i=1}^{j} \kappa_{2 i-1} \prod_{i=j+1}^{\frac{n-3}{2}} \kappa_{2 i}\right)^{2} \epsilon_{2 j}\right]}{\kappa_{1} \kappa_{2} \kappa_{3} \ldots \kappa_{n-2}} \kappa_{n-1}+\frac{1}{\epsilon_{1} \epsilon_{2} \ldots \epsilon_{n-1}} \frac{\kappa_{1} \kappa_{3} \ldots \kappa_{n-2}}{\kappa_{2} \kappa_{4} \ldots \kappa_{n-1}}=0
$$

Equation (4.19) equals to

$$
\frac{1}{\epsilon_{1} \epsilon_{2} \ldots \epsilon_{n-2}} \frac{\sum_{j=1}^{\frac{n-3}{2}}\left[\left(\prod_{i=1}^{j} \kappa_{2 i-1} \prod_{i=j+1}^{\frac{n-1}{2}} \kappa_{2 i}\right)^{2} \epsilon_{2 j}\right]}{\kappa_{1} \kappa_{2} \kappa_{3} \ldots \kappa_{n-1}}+\frac{1}{\epsilon_{1} \epsilon_{2} \ldots \epsilon_{n-1}} \frac{\left(\kappa_{1} \kappa_{3} \ldots \kappa_{n-2}\right)^{2}}{\kappa_{1} \kappa_{2} \ldots \kappa_{n-2} \kappa_{n-1}}=0 .
$$

A contradiction occurs again with equation (4.20). Therefore, utilizing Theorem 4.1, the proof is completed. 
Remark 4.1. Let $\beta: I \rightarrow \mathbb{E}_{1}^{n}$ be a curve with constant curvatures, parametrized by

$$
\beta(t)=\left(a_{1} \cosh \left(b_{1} t\right), a_{1} \sinh \left(b_{1} t\right), a_{2} \cos \left(b_{2} t\right), a_{2} \sin \left(b_{2} t\right), \ldots, a_{m} \cos \left(b_{m} t\right), a_{m} \sin \left(b_{m} t\right)\right),
$$

for even $n=2 m$ and

$$
\beta(t)=\left(a_{1} \cosh \left(b_{1} t\right), a_{1} \sinh \left(b_{1} t\right), a_{2} \cos \left(b_{1} t\right), a_{2} \sin \left(b_{1} t\right), \ldots, a_{m} \cos \left(b_{m} t\right), a_{m} \sin \left(b_{m} t\right), a t\right),
$$

for odd $n=2 m+1$, where $a, a_{i}, b_{i} \in \mathbb{R}$ and $b_{i}$ 's are distinct numbers for $i \in\{1,2, \ldots, m\}$.

It is clear to show that, from equations (4.21), (4.22) a curve with constant curvatures is not a spacelike rectifying curve, so

$$
\langle\beta(t), N(t)\rangle \neq 0 .
$$

Assuming all the curvatures of the spacelike rectifying curve are non-zero constants, except one of these, the non-constant curvature can be defined as follows:

Theorem 4.3. Let $\alpha: I \rightarrow \mathbb{E}_{1}^{n}$ be an arclength parametrized spacelike curve with non-zero curvatures. If the first $n-2$ curvatures $\kappa_{1}, \kappa_{2}, \ldots, \kappa_{n-2}$ of the spacelike rectifying curve $\alpha$ are non-zero constants, then, $\alpha$ is a spacelike rectifying curve if and only if

$$
\begin{aligned}
\kappa_{n-1} & = \pm \frac{1}{\sqrt{\epsilon_{n-2} \epsilon_{n-1} a s(s+2 c)+b}}, \text { for even } n, \\
\kappa_{n-1} & = \pm \frac{s+c}{\sqrt{\epsilon_{n-2} \epsilon_{n-1} a s(s+2 c)+b}}, \text { for odd } n,
\end{aligned}
$$

where $a$ is a constant allied with the curvatures $\kappa_{1}, \kappa_{2}, \ldots, \kappa_{n-2}$ and $b, c \in \mathbb{R}$.

Proof. $(\Rightarrow)$ Suppose that $\alpha$ is a spacelike rectifying curve with its first $n-2$ non-zero constant curvatures. From equation (4.5), we have

$$
\mu_{n-3} \kappa_{n-1}=-\mu_{n-2}^{\prime}=-\left(\frac{\mu_{n-4} \kappa_{n-2}+\mu_{n-3}^{\prime}}{\epsilon_{n-2} \epsilon_{n-1} \kappa_{n-1}}\right)^{\prime} .
$$

Equations (4.14), (4.15) are valid for $i \in\{1,2, \ldots, n-3\}$. Hence, for even $n$, equation (4.25) reduces to

$$
a(s+c) \kappa_{n-1}=\left(\frac{1}{\epsilon_{n-2} \epsilon_{n-1} \kappa_{n-1}}\right)^{\prime},
$$

where $a$ is a constant allied with the curvatures $\kappa_{1}, \kappa_{2}, \ldots, \kappa_{n-2}$. Equation (4.26) leads to the relation in (4.23).

In case $n$ is odd, from (4.25), we get

$$
a \kappa_{n-1}=\left(\frac{s+c}{\epsilon_{n-2} \epsilon_{n-1} \kappa_{n-1}}\right)^{\prime}
$$

where $a$ is a constant allied with the curvatures $\kappa_{1}, \kappa_{2}, \ldots, \kappa_{n-2}$. Equation (4.27) equals to

$$
\frac{s+c}{\kappa_{n-1}}\left(\frac{s+c}{\epsilon_{n-2} \epsilon_{n-1} \kappa_{n-1}}\right)^{\prime}=a(s+c),
$$

from which the solution as in the statement of the theorem follows.

$(\Leftarrow)$ Suppose that $\alpha$ is the spacelike rectifying curve with its first $n-2$ constant curvatures with $n \geq 4$ and the last curvature of $\alpha$ is defined as (4.23), (4.24). Then, the statement of the Theorem 4.1, that is equation (4.13), is

$$
\kappa_{n-1} \mu_{n-3,0}+\mu_{n-2,0}^{\prime}=0 .
$$

From system (4.12), (4.28) equals to

$$
\kappa_{n-1} \mu_{n-3,0}+\left(\frac{\kappa_{n-2} \mu_{n-4,0}+\mu_{n-3,0}^{\prime}}{\epsilon_{n-2} \epsilon_{n-1} \kappa_{n-1}}\right)^{\prime}=0 .
$$

Also, from (4.11) and the constancy of $\kappa_{1}$ and $\kappa_{2}$, (4.29) equals to (4.25). Then, the curvatures in (4.23) and (4.24) satisfy (4.25), (4.13) is completed; so, $\alpha$ is a spacelike rectifying curve. 


\subsection{The components of the position vector of a spacelike rectifying curve}

In the theorem below, a spacelike rectifying curve in $\mathbb{E}_{1}^{n}$ is characterized by its tangential component, its normal component or its binormal components.

Theorem 4.4. $\alpha$ is a spacelike rectifying curve parametrized by the arclength in $\mathbb{E}_{1}^{n}$ with non-zero curvatures iff one of the following expressions hold.

[i] The tangential component of the position vector of the spacelike rectifying curve $\alpha$ is given by

$$
\langle\alpha, T\rangle=s+c,
$$

for some constant $c \in \mathbb{R}$.

[ii] The distance function $\rho=\|\alpha\|$ provides

$$
\rho^{2}=s^{2}+c_{1} s+c_{2}
$$

for some $c_{1}, c_{2} \in \mathbb{R}$.

[iii] The normal component $\alpha^{N}$ of the position vector of the spacelike rectifying curve $\alpha$ has constant length and the distance function $\rho$ is non-constant.

[iv] The binormal components of the position vector of the spacelike rectifying curve $\alpha$ are given by

$$
\left\langle\alpha, B_{i}\right\rangle=\mu_{i},
$$

where $\mu_{i}$ for $i \in\{1,2, \ldots, n-2\}$ is defined by (4.9), (4.10) and (4.11).

Proof.

[i] $(\Rightarrow)$ Suppose that, $\alpha$ is a spacelike rectifying curve parametrized by the arclength. From the definition of $\alpha$ in (2.2) and equation (4.1), we get

$$
\langle\alpha, T\rangle=\lambda=s+c .
$$

$(\Leftarrow)$ If $\langle\alpha, T\rangle=s+c$, then differentiating this equation using the Frenet equations in (2.1), we get

$$
\langle\alpha, N\rangle \kappa_{1}=0 .
$$

Since $\kappa_{1} \neq 0, \alpha$ is a spacelike rectifying curve.

[ii] $(\Rightarrow)$ Suppose that, $\alpha$ is a spacelike rectifying curve parametrized by the arclength. Multiplying (4.3) with $\mu_{1}$, (4.4) with $\epsilon_{2} \epsilon_{i+1} \mu_{i}, i \in\{2,3, \ldots, n-3\}$ and (4.5) with $\epsilon_{2} \epsilon_{n-1} \mu_{n-2}$, we get

$$
\begin{gathered}
\mu_{1}\left(\mu_{1}^{\prime}-\epsilon_{2} \epsilon_{3} \mu_{2} \kappa_{3}\right)=0, \\
\epsilon_{2} \epsilon_{i+1} \mu_{i}\left(\mu_{i}^{\prime}+\mu_{i-1} \kappa_{i+1}-\epsilon_{i+1} \epsilon_{i+2} \mu_{i+1} \kappa_{i+2}\right)=0, \\
\epsilon_{2} \epsilon_{n-1} \mu_{n-2}\left(\mu_{n-2}^{\prime}+\mu_{n-3} \kappa_{n-1}\right)=0 .
\end{gathered}
$$

Adding equations (4.30), (4.31), (4.32), we have

$$
\mu_{1} \mu_{1}^{\prime}+\epsilon_{2} \epsilon_{3} \mu_{2} \mu_{2}^{\prime}+\epsilon_{2} \epsilon_{4} \mu_{3} \mu_{3}^{\prime}+\ldots+\epsilon_{2} \epsilon_{n-2} \mu_{n-3} \mu_{n-3}^{\prime}+\epsilon_{2} \epsilon_{n-1} \mu_{n-2} \mu_{n-2}^{\prime}=0,
$$

or

$$
\epsilon_{2} \mu_{1} \mu_{1}^{\prime}+\epsilon_{3} \mu_{2} \mu_{2}^{\prime}+\ldots+\epsilon_{n-2} \mu_{n-3} \mu_{n-3}^{\prime}+\epsilon_{n-1} \mu_{n-2} \mu_{n-2}^{\prime}=0 .
$$

Hence, we can write

$$
\sum_{i=1}^{n-2} \epsilon_{i+1} \mu_{i} \mu_{i}^{\prime}=0 .
$$

So, we have

$$
\sum_{i=1}^{n-2} \epsilon_{i+1} \mu_{i}^{2}=a^{2}
$$


for $a \in \mathbb{R}$. From the definition of $\alpha$ in (2.2) and using (4.1), we get

$$
\begin{aligned}
\rho^{2} & =\langle\alpha, \alpha\rangle=\lambda^{2}+\sum_{i=1}^{n-2} \mu_{i}^{2} \\
& =(s+c)^{2}+d^{2} .
\end{aligned}
$$

$(\Leftarrow)$ Differentiating $\rho^{2}=\langle\alpha, \alpha\rangle=s^{2}+c_{1} s+c_{2}$ twice using Frenet equations in (2.1), we obtain

$$
\langle\alpha, N\rangle=0 .
$$

Thus, $\alpha$ is congruent to a spacelike rectifying curve.

[iii] $(\Rightarrow)$ Decomposing the position vector of a spacelike curve $\alpha$ in its tangential and normal component, we come across with

$$
\alpha=\langle\alpha, T\rangle T+\alpha^{N} .
$$

From (2.2), for a spacelike rectifying curve, $\alpha^{N}=\sum_{i=1}^{n-2} \mu_{i} B_{i}$. So,

$$
\left\|\alpha^{N}\right\|=\sqrt{\sum_{i=1}^{n-2} \mu_{i}^{2}}=d,
$$

where $d$ is given in equation (4.33). Therefore, the normal component of $\alpha$ has constant length. The proof of the distance function mentioned, is given in $[i i]$.

$(\Leftarrow)$ From $\alpha^{N}=\alpha-\langle\alpha, T\rangle T$ and $\left\langle\alpha^{N}, \alpha^{N}\right\rangle=d^{2}$, we find that

$$
d^{2}=\left\langle\alpha^{N}, \alpha^{N}\right\rangle=\langle\alpha, \alpha\rangle-\langle\alpha, T\rangle^{2} .
$$

Differentiating (4.34) and using (2.1), we have

$$
\kappa_{1}\langle\alpha, T\rangle\langle\alpha, N\rangle=0
$$

Since, $\rho^{2}=\|\alpha\|^{2}$ is not a constant, $\langle\alpha, T\rangle \neq 0$. So, $\alpha$ is congruent to a spacelike rectifying curve.

[iv] $(\Rightarrow)$ It is clear from equation (2.2) for the position vector of a spacelike rectifying curve.

$(\Leftarrow)$ Suppose $\left\langle\alpha, B_{1}\right\rangle=\mu_{1}$. Differentiating and using (2.2), we obtain

$$
-\epsilon_{1} \epsilon_{2} \kappa_{2}\langle\alpha, N\rangle+\kappa_{3} \mu_{2}=\mu_{1}^{\prime} .
$$

Using the definitions of $\mu_{1}$ and $\mu_{2},\langle\alpha, N\rangle=0$. Hence, $\alpha$ is congruent to a spacelike rectifying curve.

\subsection{A classification of spacelike rectifying curves}

In this section, we build the spacelike rectifying curves starting from an arclength parametrized curve on the pseudo-hyperbolic space, $\mathbb{H}_{0}^{n-1}(1)=\left\{\vec{x} \in \mathbb{E}_{1}^{n} \mid\langle\vec{x}, \vec{x}\rangle=-1\right\}$.

Theorem 4.5. Let $\alpha: I \rightarrow \mathbb{E}_{1}^{n}$ be a spacelike curve given by $\vec{\alpha}(t)=\rho(t) \vec{y}(t)$, where $\rho(t)$ is an arbitrary positive function and $\vec{y}(t)$ is a unit spacelike curve lying in pseudo-hyperbolic space $\mathbb{H}_{0}^{n-1}(1)$. Then, $\vec{\alpha}$ is a spacelike rectifying curve if and only if

$$
\rho(t)=\frac{a}{\cosh \left(t+t_{0}\right)}, a \in \mathbb{R}_{0}, t \in \mathbb{R} .
$$

Proof. We can prove this idea with the same method used in [1].

Example 4.1. Let us consider the curve,

$$
\begin{gathered}
\vec{\alpha}(s)=\frac{a}{\cosh \left(s+s_{0}\right)} \quad\left(\sqrt{\frac{n}{2}} \cosh \left(\frac{s}{\sqrt{n-1}}\right), \sqrt{\frac{n}{2}} \sinh \left(\frac{s}{\sqrt{n-1}}\right), \sin \left(\frac{s}{\sqrt{n-1}}\right),\right. \\
\left.\cos \left(\frac{s}{\sqrt{n-1}}\right), \quad \ldots, \sin \left(\frac{s}{\sqrt{n-1}}\right), \cos \left(\frac{s}{\sqrt{n-1}}\right)\right),
\end{gathered}
$$


where $a \in \mathbb{R}_{0}, s_{0} \in \mathbb{R}$ in $\mathbb{E}_{1}^{n}$. This curve has a form $\vec{\alpha}(s)=\rho(s) \vec{y}(s)$ where $\rho(s)=\frac{a}{\cosh \left(s+s_{0}\right)}$ and

$$
\begin{aligned}
\vec{y}(s) & =\left(\sqrt{\frac{n}{2}} \cosh \left(\frac{s}{\sqrt{n-1}}\right), \sqrt{\frac{n}{2}} \sinh \left(\frac{s}{\sqrt{n-1}}\right), \sin \left(\frac{s}{\sqrt{n-1}}\right), \cos \left(\frac{s}{\sqrt{n-1}}\right),\right. \\
& \left.\ldots \quad, \sin \left(\frac{s}{\sqrt{n-1}}\right), \cos \left(\frac{s}{\sqrt{n-1}}\right)\right) .
\end{aligned}
$$

Since $\langle\vec{y}(s), \vec{y}(s)\rangle=-1$ and $\left\|y^{\prime}(s)\right\|=1, \vec{y}(s)$ is a unit speed spacelike curve in pseudo-hyperbolic space $\mathbb{H}_{0}^{n-1}(1)$. So, $\vec{\alpha}$ is a spacelike rectifying curve lying in $\mathbb{E}_{1}^{n}$.

Corollary 4.1. The statements of this paper coincide with the spacelike cases of [9] for $n=3$, and with [1] for $n=4$.

Remark 4.2. For the timelike rectifying curve, we get the following equation system

$$
\begin{aligned}
\lambda^{\prime} & =1, \\
\lambda \kappa_{1}-\mu_{1} \kappa_{2} & =0, \\
\mu_{1}^{\prime}-\mu_{2} \kappa_{3} & =0, \\
\mu_{i}^{\prime}+\mu_{i-1} \kappa_{i+1}-\mu_{i+1} \kappa_{i+2} & =0 \\
\mu_{n-2}^{\prime}+\mu_{n-3} \kappa_{n-1} & =0 .
\end{aligned}
$$

It can be clearly seen that the above system is equal to the system of [2].

\section{Conclusion}

This work gives rectifying curves and develops their characterization in Lorentz $n$-space. So, this study may shed light on future work about rectifying curves with respect to their causel character and help some unsolved problems of rectifying curves in such spaces.

\section{References}

[1] Ali AT, Önder M., Some characterizations of space-like rectifying curves in the Minkowski space-time. Glob J Sci Front Res Math Decision Sci, 12(1) (2012), 57-64.

[2] Cambie S, Goemans W and Van Den Bussche I., Rectifying curves in n-dimensional Euclidean space. Turk J Math , 40 (2016), $210-223$.

[3] Chen B-Y., When does the position vector of a space curve always lie in its rectifying plane? Am Math Mon, 110 (2003), 147-152.

[4] Chen B-Y, Dillen F. Rectifying curves as centrodes and extremal curves. Bull Inst Math Acad Sinica, 33 (2005), 77-90.

[5] İlarslan K, Nešović E., Some characterizations of null, pseudo null and partially null rectifying curves in Minkowski space-time. Taiwanese J Math, 12(5) (2008), 1035-1044.

[6] İlarslan K. and Nešović E., Some characterizations of rectifying curves in the Euclidean space $\mathbb{E}^{4}$. Turk J Math, 32 (2008), $21-30$.

[7] İlarslan K. and Nešović E., On rectifying curves as centodes and extremal curves in the Minkowski 3-space. Novi Sad J Math, 37 (2007), 53-64.

[8] İlarslan K. Spacelike normal curves in Minkowski space $\mathbb{E}_{1}^{3}$. Turk J Math, 29 (2005), 53-63.

[9] İlarslan K., Nešović E, Petrović-Torgašev M., Some characterizations of rectifying curves in the Minkowski 3-space. Novi Sad J Math, 33 (2003), 23-32.

[10] İlarslan K., Some special curves on non-Euclidean manifolds. PhD, Ankara University, Ankara, Turkey, 2002.

[11] Izumiya S, Takeuchi N., New special curves and developable surfaces. Turk J Math, 28 (2004),153-163.

[12] Lucas P, Ortega-Yagües JA., Rectifying curves in the three-dimensional sphere. J Math Anal Appl, 421 (2015), 4855-4868.

[13] O'neill B. Semi-Riemann Geometry with application to relativity. New York: Academic Press, 1983.

[14] Öztekin H, Öğrenmiş AO. Normal and rectifying curves in Pseudo-Galilean space $\mathbb{G}_{1}^{3}$ and their characterizations. J Math Comput Sci, 2 (2012), 91-100.

[15] Yücesan, A., Ayyıldız, N., Çöken, A. C., On Rectifying Dual Space Curves. Revista Matematica Complutense, 20(2) (2007), 497-506. 


\section{Affiliations}

TUNAHAN TURHAN

ADDRESS: Vocational School of Technical Sciences, Süleyman Demirel University,

32260, Isparta, Turkey

E-MAIL: tunahanturhan@sdu.edu.tr

ORCID ID: orcid.org/0000-0002-9632-2180 